E Research Square

\title{
The Brain Is Globally Symmetric: An Analysis of Intra and Interhemispheric Symmetry of Subcortical Structures in the Aging Human Brain
}

Jaime Gomez Ramirez ( $\sim$ jd.gomezramirez@gmail.com )

Instituto de Salud Carlos III https://orcid.org/0000-0002-9440-7711

Javier J González-Rosa

Universidad de Cadiz

\section{Research Article}

Keywords: MRI, Hemispheric asymmetry, Laterality, Aging, Eigenvalues

Posted Date: March 11th, 2021

DOI: https://doi.org/10.21203/rs.3.rs-267371/v1

License: (c) (i) This work is licensed under a Creative Commons Attribution 4.0 International License.

Read Full License 


\title{
The brain is globally symmetric: an analysis of intra and interhemispheric symmetry of subcortical structures in the aging human brain
}

\author{
Jaime Gómez-Ramírez ${ }^{1,3}$ and Javier J. González-Rosa² \\ ${ }^{1}$ Instituto de Salud Carlos III \\ ${ }^{3}$ Instituto de Investigación Sanitaria Hospital Clínico San Carlos (IdISSC) Madrid, \\ Spain \\ ${ }^{2}$ Department of Psychology, Universidad de Cádiz, Spain. , Instituto de \\ Investigación Biomédica de Cádiz (INIBICA)
}

\begin{abstract}
Here we address the hemispheric interdependency of subcortical structures in the aging human brain. In particular, we investigate whether volume variation can be explained with the adjacency of structures in the same hemisphere or is due to the interhemispheric development of mirror subcortical structures in the brain. Seven subcortical structures in both hemispheres were automatically segmented in a large sample of over three 3,312 magnetic resonance imaging (MRI) studies of elderly individuals in their 70s and 80s. We perform Eigenvalue analysis to find that anatomic volumes in the limbic system and basal ganglia show similar statistical dependency when considered in the same hemisphere (intrahemispheric) or in different hemispheres (interhemispheric). Our results indicate that anatomic bilaterality is preserved in the aging human brain, supporting the hypothesis that coupling between non-adjacent brain areas could act as a mechanism to compensate for the deleterious effects of aging.
\end{abstract}

Keywords: MRI; Hemispheric asymmetry; Laterality; Aging; Eigenvalues

\section{INTRODUCTION}

The interest and use of understanding the differences between the two brain hemispheres go back a long way [Lashley, 1958], [Sperry, 1984], [LeDoux et al., 2020]. Two of the most revelatory discoveries are the Broca area and Sperry split-brain experiments. Broca found that a patient that could only utter the syllable "tan" had a large lesion in the left posterior inferior frontal gyrus that was subsequently named the Broca's area [Ocklenburg and Gunturkun, 2012]. Hemispheric asymmetry and its importance in cognition acquired a dramatic turn with Sperry's split-brain experiments in cats, monkeys, and later on epileptic patients. Sperry showed that disconnecting the two hemispheres by severing the corpus callosum resulted in making the two hemispheres functionally independent [Sperry, 1961]. 
However, and as it could not be otherwise given the staggering complexity of the brain, the lateralizations and anatomic asymmetries can not be accounted for within a simple narrative, for example, the left-right dominance [Nielsen et al., 2013], conclusions may vary depending on the methodology applied and the brain region or systems of interest. For instance, the increasing availability of data from volumetric brain imaging has made possible to study the effect of lateralized functions on subcortical asymmetries [Morillon et al., 2010], [Kang et al., 2015], [Rane et al., 2017], [Narvacan et al., 2017], [Núñez et al., 2018], [Esteves et al., 2019] and to postulate lateralization alterations as potential endophenotypic markers in chronic brain disorders such as schizophrenia [Dennison et al., 2013], [Roalf et al., 2015], |Okada et al., 2018] and other brain conditions, including Alzheimer's disease [Giannakopoulos et al., 1994], dyslexia [Leonard and Eckert, 2008] and autism [Floris et al., 2020].

Evolutionary biology explains lateralization in the brain as a trade-off between bilateral symmetry and hemispheric asymmetry to cope with the external world. As suggested by Palmer [Palmer, 2004], bilateral symmetry may be the default condition, noticing that the mid-plane of developing organisms has anterior-posterior and dorso-ventral axes but there is no left-right axis. In a world where predators may come from either side -right and left- sensory asymmetry would bring in the high price of being more vulnerable to predators approaching from the weak side. Along these lines, bilateral symmetry could be the result of natural adaptation. Nevertheless, the brain and other organs count with left-right asymmetries, such asymmetries could have evolved when proving to be more adaptive to the environment. For example, bilateral symmetry in limbs and legs is adaptive because it can produce linear movement. Thus, directional locomotion impinges a front-back asymmetry conserving the left-right symmetry.

Sensorimotor processing in the brain is overall organized symmetrically, however asymmetries such as handedness are compatible with the cerebral organization [Corballis, 2009], [Willems et al., 2010], [Neubauer et al., 2020]. Whether intrahemispheric coupling is more predominant than bilateral symmetry, or lateralized interhemispheric components are more tightly coupled than intrahemispheric components, is still poorly understood [van der Knaap and van der Ham, 2011].

Automatic segmentation of subcortical structures can produce important insights regarding the anatomic symmetric organization of the brain [Hervé et al., 2013], |Kong et al., 2020]. Subcortical structures are groups of neural formations deep within the brain, among these structures we find the limbic structures and the basal ganglia. The basal ganglia subcortical nuclei -caudate, putamen, pallidum- are located near the thalamus which is part of the limbic system. The limbic system refers to a group of subcortical nuclei -hypothalamus, thalamus, amygdala, hippocampus, accumbens- that supports a large variety of functions and behaviors such as long-term memory and affective responses [Shaw and Alvord Jr, 1997], [McLachlan, 2009]. The subcortical nuclei that compose the basal ganglia support motor control but they are also involved in motor learning, executive actions and affective responses [Albin et al., 1989], [Kreitzer and Malenka, 2008]. Agerelated changes of limbic system in normal population have been shown using different imaging techniques, diffusion tensor imaging [Gunbey et al., 2014] and magnetic resonance imaging [Callen et al., 2001], [Fjell et al., 2015]. However, a systematic and quantitative assessment, based on a large sample, of global symmetry in subcortical brain structures in the elderly brain is still missing.

In this study we perform volumetric segmentation in over 3,000 magnetic resonance imaging (MRI) studies in healthy elderly subjects, obtaining the volumetric estimation of the following seven subcortical structures: caudate, pallidum, putamen, thalamus, hippocampus, amygdala, and accumbens. Both global and lateralized subcortical brain symmetry are quantitatively assessed using correlation matrices and Eigenvalue analysis.

The rationale of selecting subcortical structures across and within hemispheres is to determine whether correlation based on locus (vicinity of structures) or genus (kind of structure) is maintained 
in the brain of elderly subjects. In particular, we investigate whether volumetric variation can be explained with adjacency of structures in the same hemisphere, or is due to interhemispheric development of mirror subcortical structures in the brain.

\section{Methodology}

The dataset used here comes from a single-center, observational cohort study [Gómez-Ramírez et al., 2020]. The participants of the study are home-dwelling elderly volunteers, aged 70 to 85 , without relevant psychiatric, neurological, or systemic disorders. Of the initial 1,213 subjects, those that were diagnosed with MCI or dementia plus those lacking a brain MRI were excluded from our analysis, resulting in a cohort of 890 healthy elderly subjects. The subjects were assessed yearly for a total of five years resulting in 3312 assessments with the number of yearly visits per subject varying from 2 to 5 .

After signing informed consent, the participants undertake a yearly systematic clinical assessment including medical history, neurological, and neuropsychological exam, blood collection, and brain MRI. APOE genotype was studied with total DNA isolated from peripheral blood following standard procedures. Ethical approval was granted by the Research Ethics Committee of Instituto de Salud Carlos III and written informed consent was obtained from all the participants. The authors assert that all procedures contributing to this work comply with the ethical standards of the relevant national and institutional committees on human experimentation, and with the Helsinki Declaration of 1975 and its later amendments.

\section{Imaging Study}

The imaging data were acquired in the sagittal plane on a 3T General Electric scanner (GE Milwaukee, WI) utilizing T1-weighted inversion recovery, supine position, flip angle 12॰, 3-D pulse sequence: echo time Min. full, time inversion $600 \mathrm{~ms}$., Receiver Bandwidth $19.23 \mathrm{kHz}$, field of view $=24.0 \mathrm{~cm}$, slice thickness $1 \mathrm{~mm}$ and Freq $\times$ Phase $288 \times 288$. The preprocessing of MRI 3 Tesla images in this study consisted of generating an isotropic brain image with nonbrain tissue removed. We used the initial, preprocessing step in the computational segmentation tool used in this study, the FreeSurfer pipeline (recon-all [FreeSurfer cortical reconstruction and parcellation process., 2017]). The postprocessing was performed with FreeSurfer [Fischl, 2012|, version freesurfer-darwin-OSX-ElCapitan-dev-20190328-6241d26 running under Mac OS X, product version 10.14.5.

FreeSurfer includes tools for processing structural MRI, functional MRI, diffusion MRI and PET data. Here we are focusing on structural MRI, in particular subcortical segmentation. The cross-sectional analysis starts with the surface-based stream [Dale et al., 1999, Fischl et al., 1999], where the skull is stripped, the cerebellum and brain stern are removed, the two hemispheres are separated and brain voxels are classified as white matter or grey matter and CSF[Fischl] et al., 2002, Fischl et al., 2004]. Then, the volume-based stream segments the different subcortical structures of the brain using a subject-independent probabilistic atlas. The FreeSurfer training set consists of 40 MRIs, spread in age (10 healthy young subjects, 10 healthy middle-aged subjects, and 10 healthy elderly subjects) and including pathological brains (10 subjects with AD) [Desikan et al., 2006.

The stages in the FreeSurfer pipeline (in order) are surface-based stream, with skull-stripping cerebellum and brain stern removal, two hemispheres separation, and brain voxels classification (white matter, gray matter, and CSF), and finally brain segmentation, cortical and subcortical. 
The subcortical segmentation includes seven structures in each hemisphere, namely, thalamus, putamen, hippocampus, caudate, pallidum, amygdala, and accumbens.

For the sake of illustration, Figure 1 shows the result produced of the intracranial volume segmentation in four subjects in the study out of a total of 3312 MRI scans.
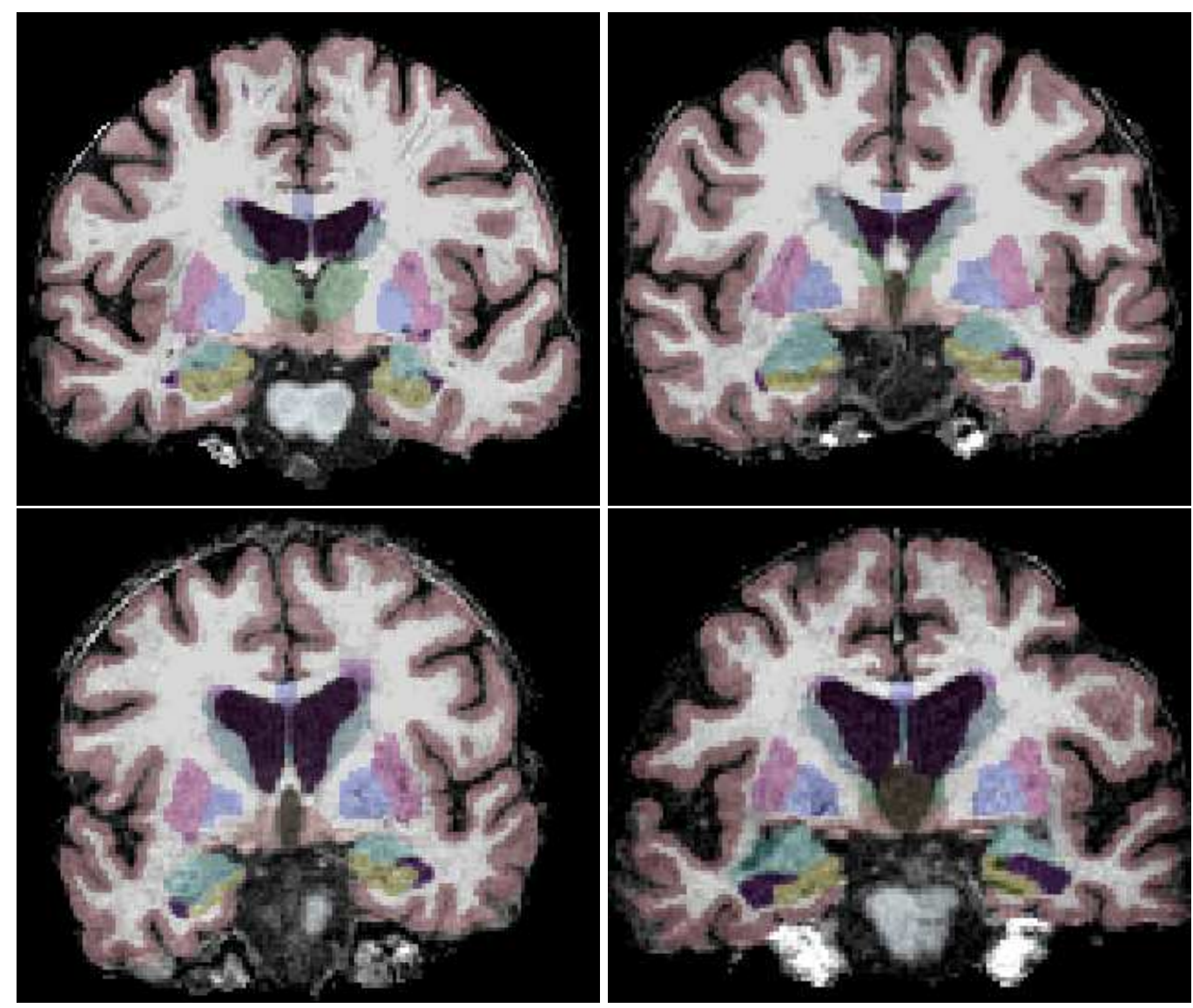

Figure 1: Subcortical structures are clusters of cell bodies, buried in white matter area. Coronal view of the subcortical segmentation realized in 4 different subjects in the study. Clockwise from top left: 75 female 75,74 male, 76 female, 83 female.

\section{Anomaly detection with the Isolation Forest Algorithm}

One premise of big data applied to brain imaging research is that given enough data, we may be able to characterize brain atrophy and fit the data to a model that makes predictions about the dynamics of the atrophy, that is, not only identify atrophy but its progression in an individual basis.

Manual segmentation of the brain is a time consuming and prone to error task [Vos et al., 2019], [Despotović et al., 2015], [Firbank et al., 2008], [Collier et al., 2003]. The segmentation of one volume may require an entire day of work from a dedicated expert. Hence, the use of manual segmentation on a dataset as ours in order of thousand of MRI scans is not an option. The main advantages of automatic procedures are at least two: i) the lack of bias inherent in manual segmentation, two different human experts may produce very different estimates for the 
same image, and ii) automatic procedures are time-saving. On the other hand, automated quality control is paramount to avoid the inclusion of inaccurate measurements in the posterior analysis. Anomaly detection [Chandola et al., 2009] is an established approach in data analysis to identify observations with suspicious statistical properties when compared to the majority of data.

Isolation Forest [Liu et al., 2008] is an ensemble method [Breiman, 1996] that has shown particularly strong performance as an outlier detector in a variety of datasets [Domingues et al., 2018],|Alaverdyan, 2019]. The algorithm works by selecting features and randomly selecting a split value between the maximum and minimum values of the selected feature. Representing partitioning with a tree structure, the number of splitting required to isolate a sample is equal to the path length from the tree's root node to the tree's leaf node. By averaging the path length over a forest of random forests we get a measure of normality, specifically, a forest with shorter path lengths for particular samples indicates that the samples are likely to be anomalies.

We perform anomaly detection using the scikit-learn Isolation Forest implementation [Pedregosa et al., 2011]. The parameters, number of estimators, number of samples, and contamination level are set to the default values.

\section{Statistical Analysis}

Table 1 shows the description of the variables included in the study: age, sex, APOE gene, handedness and the volumetric estimates of the subcortical structures for a total of $3312 \mathrm{MRI}$ studies.

\begin{tabular}{|c|c|c|c|c|c|c|c|c|}
\hline \multicolumn{3}{|c|}{$N=3312$} & \multicolumn{3}{|c|}{$\mu+\sigma$} & \multicolumn{3}{|c|}{ groups } \\
\hline \multicolumn{3}{|c|}{ Age } & $76.51+0$ & & & & & \\
\hline \multicolumn{3}{|c|}{ Sex } & & & & $\mathrm{F}, \mathrm{N}$ & 42128,11 & \\
\hline \multicolumn{3}{|c|}{ APOE } & & $\epsilon(23)$ & $(23), \epsilon(2$ & 3) $\epsilon 4, \epsilon 4 \epsilon 4$ & 2790,502 & \\
\hline \multicolumn{3}{|c|}{ Handedness } & & & & $\mathrm{R}, \mathrm{L}, \mathrm{A}$ & 3139,89, & \\
\hline Vol. $(\mathrm{mm}$ & $\left.n^{3}\right)$ & L_Thal & L_Puta & L_Amyg & L_Pall & L_Caud & L_Hipp & L_Accu \\
\hline mean & & 5946.3 & 3917.0 & 1252.6 & 1700.8 & 3202.9 & 3527.2 & 457.1 \\
\hline std & & 615.6 & 470.2 & 187.5 & 235.6 & 438.4 & 373.1 & 87.7 \\
\hline Vol. $\left(\mathrm{mm}^{3}\right.$ & & R_Thal & R_Puta & R_Amyg & R_Pall & R_Caud & R_Hipp & R_Accu \\
\hline mean & & 5798.5 & 3952.2 & 1399.3 & 1632.8 & 3379.0 & 3608.5 & 459.1 \\
\hline std & & 554.3 & 468.6 & 204.7 & 228.9 & 470.3 & 400.4 & 85.4 \\
\hline
\end{tabular}

Table 1: The table shows the description of the variables included in the study. On top, the age -mean and standard deviation of the distribution- followed by the sex of the participants $-64 \%$ of women- the Apolipoprotein $\epsilon 4-\epsilon 2,3 \epsilon 2,3(84.2 \%), \epsilon 3 \epsilon 4$ (15.2\%), $\epsilon 4 \epsilon 4$ (0.6\%)- and the handedness -right handed (95\%), left handed (2.6\%) and ambidextrous (2.4\%). Bellow, the tables with the mean and standard deviation of the volumetric segmentation for each of the 7 structures for each hemisphere.

To investigate whether the conditions -sex, handedness and APOE- can explain the variance in the volumetric estimate of the difference between the left hemisphere (LH) and the right hemisphere (RH), we build a regression model for each subcortical structure and condition as shown in Equation 1. Thus, we split the total variation of the dependent variable, the difference between the left and right hemisphere volume of each subcortical structure, into sources of 


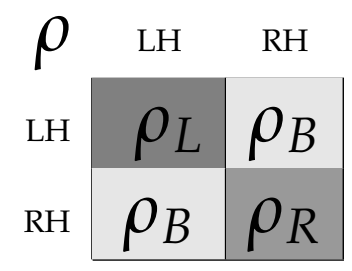

Table 2: Decomposition of correlation matrix into three submatrices, $\rho_{L}$ selects pair of structures in the left hemisphere, $\rho_{R}$ is the selection of pair of structures in the right hemisphere, and $\rho_{B}$ is the correlation matrix of pair of structures located in different hemispheres.

variation in order to find out whether the independent variable (sex, APOE, handedness) has a significant effect on the dependent variable (interhemispheric volumetric difference of subcortical structures).

$$
S_{D}=S_{L}-S_{R} \sim C(X)+C(H)+C(X)
$$

where $S_{L}$ is the volume of the LH subcortical structure $S, S_{R}$ is the volume of the RH subcortical structure $S$ and $S_{D}$ is the volumetric normalized difference between the two hemispheres in structure $S$. $X, H$ and $A$ represent the variables sex, handedness and APOE respectively codified as categorical.

\section{Aggregate correlation analysis}

The correlation matrix describes the degree to which any pair of two random variables in a set of random variables tend to deviate from their expected values. Thus, the element $i, j$ in the correlation matrix $M, M_{i, j}$ contains the correlation coefficient between the $i$ - th random variable and the $j-t h$ one. The correlation matrix can be computed from the covariance matrix if we are interested in both the strength and direction of the linear relationship between any pair of variables in the dataset. The correlation matrix is thus a collection of correlation coefficients expressing the standardized covariance between variables in a dataset.

To study whether statistical dependence between structures can be explained based on contiguity of structures (intrahemispheric) or in terms of the bilateral development of the brain (interhemispheric), we extract from the correlation matrix $\rho$ that contains the correlation coefficients between all variables, three correlation matrices, two intrahemispheric and one interhemispheric. Accordingly, from $\rho$, we obtain $\rho_{R}$ or the correlation matrix of the structures located in the right hemisphere, $\rho_{L}$ or the correlation matrix of the structures in the left hemisphere, and the bilateral correlation matrix $\rho_{B}$ which contains the correlation between any pair of structures as long as they are in different hemispheres. All three matrices $\rho_{L}, \rho_{B}$, and $\rho_{B}$ are $7 x 7$ dimensional and correlation matrix $\rho$ is $14 \times 14$ dimensional. This is shown schematically in Table 2 .

A first approximation to assess the overall strength of a correlation matrix could be done by averaging all the correlation coefficients, however, the average correlation will be biased and the average will tend to underestimate the true correlation. The Fisher transform can overcome this problem and yield an unbiased estimate by performing the $\mathrm{Z}$ transform of the correlation matrix, and then inverse transform the average. Formally, given the correlation coefficient $r$ between two variables, the Fisher's z-transform of $\mathrm{r}$ and the inverse transform is as follows: 
Fisher z-transform:

$$
\begin{aligned}
& z=\frac{1}{2} \ln \left(\frac{1+r}{1-r}\right)=\arctan (r) \\
& r=\frac{e^{2 z}-1}{e^{2 z}+1}=\tanh (z)
\end{aligned}
$$

Accordingly, the total correlation of the intrahemispheric correlation matrices $\left(\rho_{L}, \rho_{R}\right)$ and the interhemispheric $\left(\rho_{B}\right)$ correlation matrix shown in 2 can be estimated using the Fisher ztransformation as shown schematically in Equation 3.

$$
\begin{aligned}
& P_{L}=\tanh \left(\operatorname{arctanh}\left(\overline{\rho_{L}}\right)\right) \\
& P_{R}=\tanh \left(\operatorname{arctanh}\left(\overline{\rho_{R}}\right)\right) \\
& P_{B}=\tanh \left(\operatorname{arctanh}\left(\overline{\rho_{B}}\right)\right)
\end{aligned}
$$

\section{Eigenvalue analysis}

Since eigenvectors and eigenvalues uniquely define the covariance matrix, we can represent the covariance matrices in 2 by its eigenvectors and eigenvalues and therefore gain an understanding of the shape of the dataset.

We can map the $n(n-1) / 2$ correlations among $\mathrm{n}$ variables contained in a correlation matrix into $n$ eigenvalues and their associated eigenvectors with the eigenvalues being linear functions of the underlying correlations. Importantly, when all correlations are positive, the first eigenvalue is approximately a linear function of the average correlation among the variables and tells us the amount of variance contained in the correlation matrix that can be accounted for with a linear model by a single underlying factor. We can naturally extend this idea to the second, third, and so on, eigenvalues. Once we have computed the eigenvalues $\lambda_{i}$ of a correlation matrix, we can compute the area defined by the cumulative eigenvalue function as an aggregate measurement of the total cumulative percentage of variance retained by each dimension (Equation 4 ).

$$
S=\int_{\lambda_{1}}^{\lambda_{n}} F(\lambda) d \lambda
$$

We can calculate the $S_{L}, S_{R}$ and $S_{B}$ for each correlation matrix shown in 2 the cumulative curve $F(\lambda)$ would indicate the independence of the variables within each matrix, that is, the degree of independence of the subcortical structures in each hemisphere $\left(S_{L}, S_{R}\right)$ and interhemispherically $\left(S_{B}\right)$.

\section{Results}

We use the Isolation Forest Algorithm [Liu et al., 2008] to remove outliers in the dataset using the sklearn implementation [Pedregosa et al., 2011]. Isolation Forest is an unsupervised learning algorithm for detecting anomalies. The algorithm explicitly isolates anomalous points in the dataset rather than detecting points that fall outside the "normal" profile. From the dataset free of anomalies detected by the algorithm, we select the cases diagnosed as healthy, that is to say, cases with diagnosis mild cognitive impairment or Alzheimer's disease are removed from the dataset to deal only with elderly healthy brains. The resulting dataset makes a total of 3,312 MRI scans of healthy brains segmented to estimate the volume of seven subcortical structures, namely Thalamus, Putamen, Amygdala, Pallidum, Caudate, Hippocampus and Nucleus Accumbens. 
Figure 2 shows the volume estimates of the subcortical structures The structure with the largest volume is the Thalamus, followed by Putamen, Hippocampus, Caudate, Pallidum, Amygdala and lastly Accumbens, the size of the same structure in either hemisphere is very similar. For example, the mean distribution of the percentage difference between the right and the left hemisphere is $-1.47 \%$ Thalamus, $0.3 \%$ Putamen, $0.8 \%$ Hippocampus, $1.76 \%$ Caudate, $1.38 \%$ Pallidum, $1.57 \%$ Amygdala and $0.02 \%$ Nucleus Accumbens. The right hemisphere volume is on average slightly larger than the left hemisphere volume for all structures except the Thalamus.

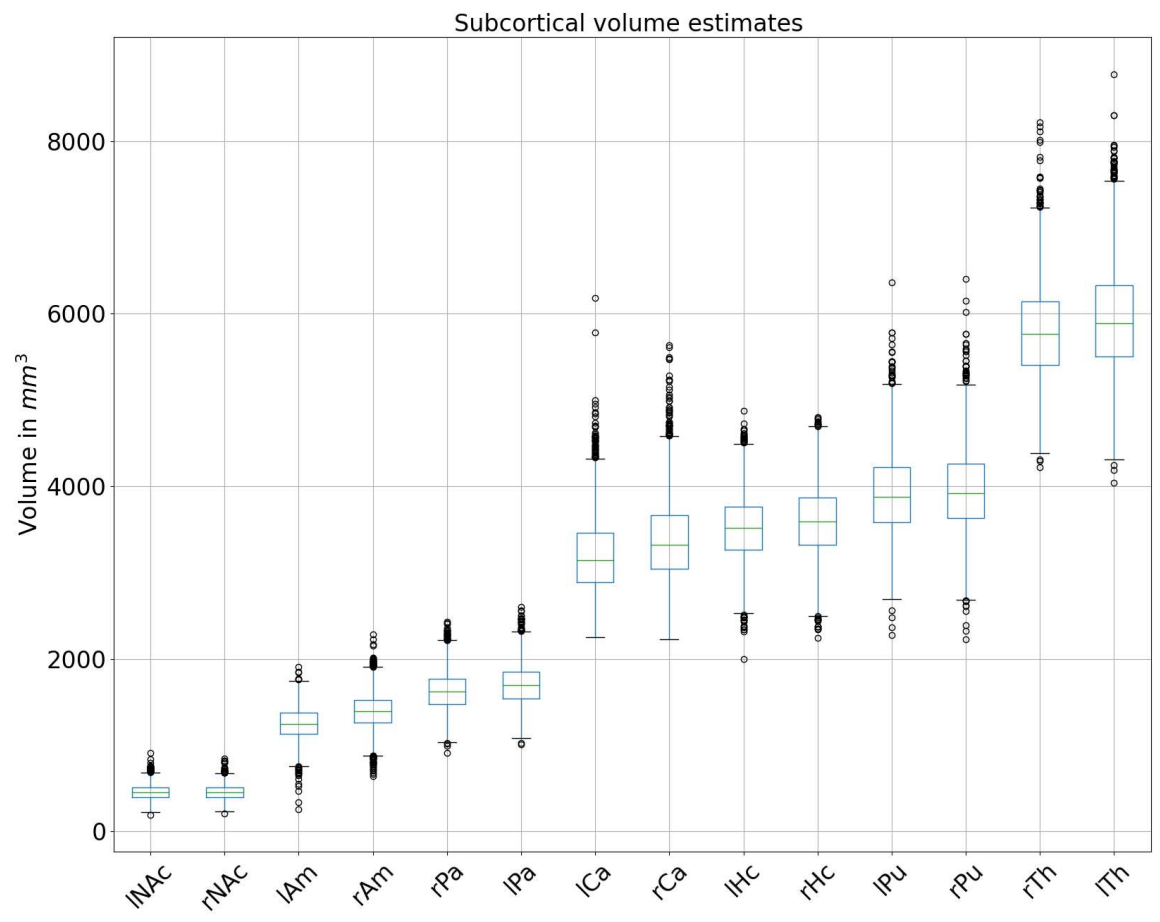

Figure 2: Boxplot of the 7 bilateral subcortical structures segmented with FreeSurfer Healthy subjects only. The estimated median volumes from the smallest to the largest structure is as follows: Accumbens R/L 459.10/457.10 $\mathrm{mm}^{3}$, Amygdala R/L 1399.31/1252.65 $\mathrm{mm}^{3}$, Pallidum R/L $1632.85 / 1700.82 \mathrm{~mm}^{3}$, Caudate R/L 3379.04/3202.93 $\mathrm{mm}^{3}$, Hippocampus R/L 3312.00/3312.00 $\mathrm{mm}^{3}$, Putamen R/L 3952.21/3916.95 $\mathrm{mm}^{3}$, Thalamus R/L 5798.49/5946.30 $\mathrm{mm}^{3}$

In the analysis presented in Table 3, we focus on the effect of sex, APOE and handedness in the preservation of symmetry for each subcortical structure, calculated as the difference between the volume for each hemisphere of the same structure (Equation 11). We fit a regression model for each bilateral structure (thalamus, putamen, amygdala, pallidum, caudate, hippocampus, caudate) and conditions sex, APOE and handedness. For the sake of simplicity we show only the from the Prob (F-statistic) from the OLS regression results. Since the data is not balanced (different sample size for each group ), we perform a type 3 sums of squares. Both type 2 and type 3 sums of squares yield similar results [Seabold and Perktold, 2010] which is due to the fact that the underlying assumption of type 2 of no interactions between factors holds [Langsrud, 2003]. 


\begin{tabular}{lccc}
\hline $\mathbf{P}($ F-statistic) & Sex & APOE & Handedness \\
Thalamus $_{D}$ & $* * *$ & & $* * *$ \\
Putamen $_{D}$ & $* * *$ & & \\
Amygdala $_{D}$ & $* * *$ & & \\
Pallidum $_{D}$ & $* * *$ & & $*$ \\
Caudate $_{D}$ & $* * *$ & & \\
Hippocampus $_{D}$ & $* * *$ & & \\
Accumbens $_{D}$ & & & \\
\hline & $\mathbf{( P}<\mathbf{0 . 0 5}^{*}$, & $\mathbf{P}<\mathbf{0 . 0 1}^{* *}$, & $\mathbf{P}<\mathbf{0 . 0 0 1}^{* * *} \mathbf{)}$ \\
\hline
\end{tabular}

Table 3: Table with the analysis of variance using the F-test to determine whether the variability between group means is larger than the variability of the observations within the groups. All the structures except the accumbens show significant bilateral volumetric difference between the two sexes $(P<<0.001)$. No structure is found to have significant differences $(p<0.01)$ among the three forms of APOE -no $\epsilon 4$ allele inherited, one $\epsilon 4$ allele, and $2 \epsilon 4$ alleles inherited from both parents. Regarding handedness, thalamus and hippocampus $(p<0.001)$ show significant differences between the three groups -right-handed, left-handed and ambidextrous. $\left({ }^{*} \mathrm{P}<0.05, * *\right.$ $\left.\mathrm{P}<0.01{ }^{* * *} \mathrm{P}<0.001\right)$

The results support the hypothesis of sex related differences in the symmetry of subcortical structures studied with the only exception of accumbens and amygdala. Studies of sex differences in the relative volume of subcortical structures have produced conflicting results with studies reporting differences in putamen, hippocampus, amygdala, thalamus and pallidum |Ruigrok et al., 2014], while [Ritchie et al., 2018] found no statistically significant differences in the hippocampus, caudate, and thalamus after adjusting for difference in total brain size between men and women. The inconsistencies found in the literature on sex differences can be motivated by the use of different types of MRI scans, segmentation algorithms, statistical analysis, size and characteristics of the sample etc |Herron et al., 2015|. The present study is single-center with identical protocol for image acquisition, image segmentation and postprocessing and is therefore impervious to those limitations. Thus, our results are relatively impervious to lack of consistent findings due to differences in the magnetic field strength of the MRI scanner, or differences in the quantification methods of segmentation. Furthermore, the potential problems of age-related changes in subcortical volume sample is reduced by addressing a large of sample of elderly subjects in their seventies and eighties.

The correlation matrix $\rho$ of the 14 structures automatically segmented is shown in Figure 3 The correlation coefficient of the same structure across the two hemispheres (adjacent cells of the main diagonal) is larger than between any two other structures. The two smallest structures, the amygdala and the nucleus accumbes are also the two structures with the weaker correlation coefficient .68 and .66 respectively, while for the rest of the structures is above 0.8 . The Accumbens happens to be also the structure that shows on average the least statistical dependence with the rest of the structures (last two rows in Figure 3). The hippocampus and the amygdala are the two structures with the strongest correlation, this holds true for the three correlation matrices, the maximum value excluded the principal diagonal is always for the Amygdala, Hippocampus pair. Regarding the correlation matrix of interhemispheric structures, the ranking of bilateral correlation in decreasing order is as follows: Caudate, Putamen, Thalamus, Hippocampus, Pallidum, Amygdala and Accumbens. Always the strongest correlation is for the bilateral structures 
$\left(\rho\left(x_{L}, x_{R}\right)>\rho\left(x_{L}, y_{R}\right), \forall y\right)$, that is, the statistical dependence between the same left and the right structure $(x)$ is larger than for any two different structures $(x, y)$.

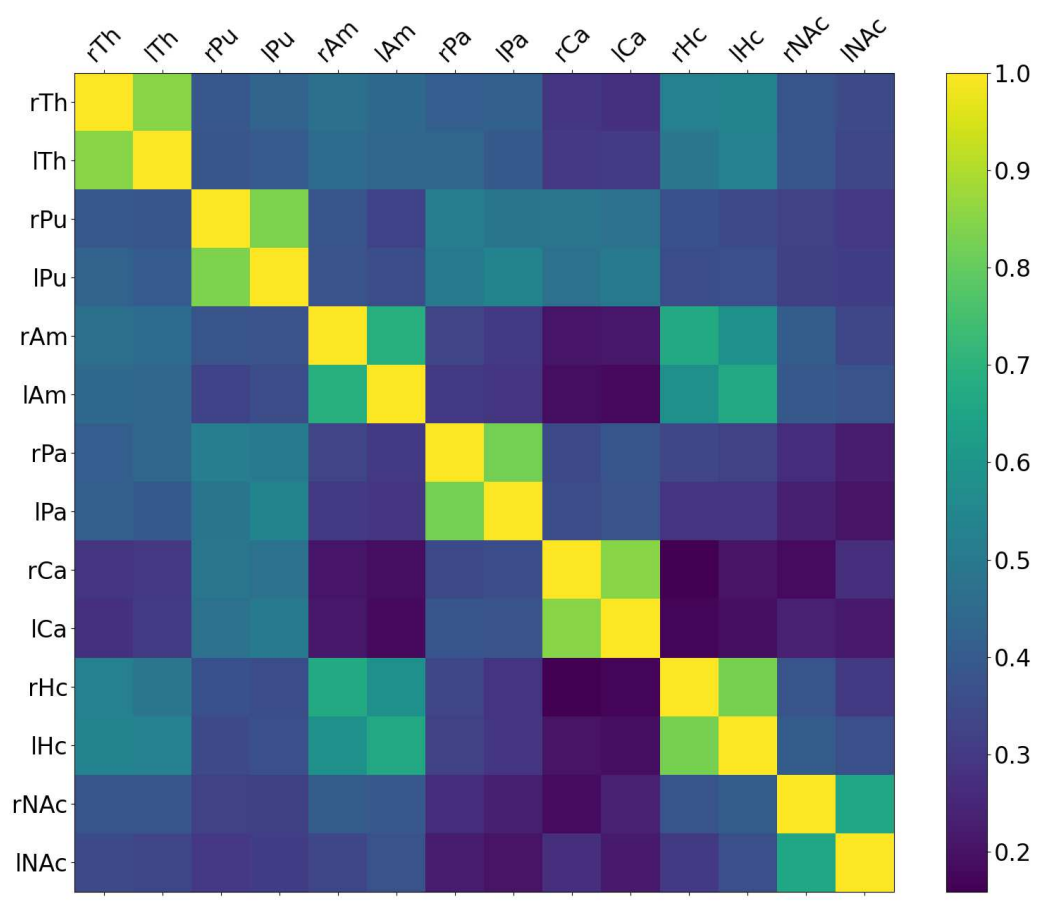

Figure 3: Correlation matrix of the 7 bilateral subcortical structures segmented with FreeSurfer. The elements of the diagonal are naturally 1 , the correlation matrix seems to indicate that the strongest statistical dependence is for the inter hemispheric correlation of the same structure.

Admittedly, the three $7 \times 7$ submatrices shown in Table 2 do not contain any additional information that is not already included in the $14 \times 14$ correlation matrix $\rho$ shown in Figure 3 The rationale of selecting structures across and within hemispheres is to acquire both intrahemispheric and interhemispheric views of the brain. By isolating the study of statistical dependence in a single hemisphere versus the entire span, we can establish whether the volume variation of the different subcortical structures is better explained locally (i.e. spatial proximity structure in the same hemisphere) or globally (i.e. connection across hemispheres).

We are, thus, interested in acquiring a systemic view of the statistical dependency among subcortical structures. To that end, we need to understand how their volumes are related according to their hemispheric location. As shown in Table 2 it is possible to extract the correlation matrices for each hemisphere and the bilateral case. However, a correlation matrix is a list of correlation coefficients, while what we need is an aggregate of the overall correlation for each matrix. As discussed in Section II we can't compute the average of a correlation matrix because correlation coefficients are not additive. However, an approximation of the overall strength of a correlation matrix can be calculated by computing the Fisher's z-transform of each coefficient $r$ in the correlation matrix, averaging the total to finally compute the inverse transform (Equation 3). The 
result of applying the Fisher's z-transform to obtain the overall correlation in the bilateral, left, and right hemisphere correlation matrices is shown in figure 4.

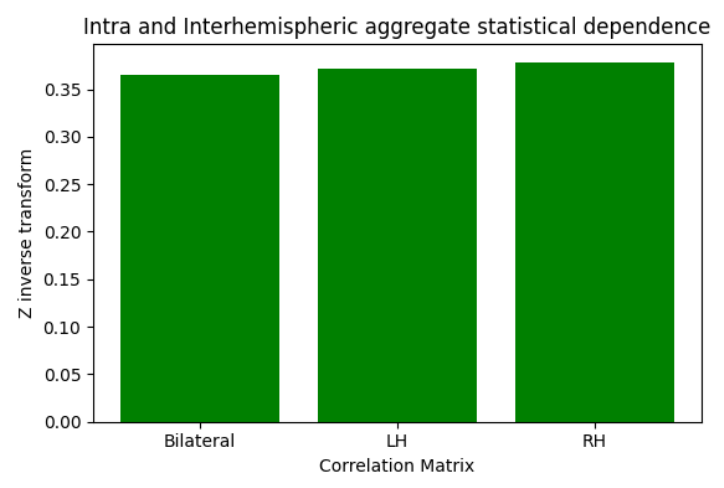

Figure 4: Aggregate statistical dependence for interhemispheric and intrahemispheric subcortical volume estimates computed with the $\mathrm{Z}$ inverse transform (Equation 3). The statistical dependence is remarkably similar in all three cases. Of note, the main diagonal has been excluded from the computation of the $\mathrm{Z}$ transform to avoid biased towards the interhemispheric matrices which symmetric and have 1 in the main diagonal which is not the case in the Bilateral correlation matrix.

We finalize our investigation of the intrahemispheric versus interhemispheric statistical dependence of subcortical brain structures with Principal Component Analysis (PCA) of the three correlation matrices $\rho_{L}, \rho_{B}$ and $\rho_{B}$ (Table 2). The eigenvectors and eigenvalues of the data covariance matrix allow us to find the principal components in order of significance. Thus, the first eigenvalue is the variance of the first principal component, the second of the second component and so on.

The distribution of the eigenvalues calculated for each correlation matrix are shown in Figure 5 Next, to assess the degree of independence of the structures volume, intra and interhemispheric, we compute the area defined under the curve defined by the cumulative eigenvectors shown in figure 5. The area under the curve described by the cumulative eigenvalues is computed using the trapezoidal integration rule i.e. dividing the total area into many trapezoids that yields the approximated area.

The area defined for the eigenvalues in the bilateral matrix is slightly larger for the interhemispheric correlation matrix, $B=.7143, L=.6795 R=.6794$. Thus, the area of the left and right hemispheres renders almost identical results, while the cumulative eigenvalues relative area for the bilateral correlation matrix is only $5 \%$ larger. Note that if the data were independent, then the cumulative curve would have an area of .5 as shown in the discontinuous red line in Figure 5 The amount of information both unilaterally and bilaterally is therefore similar. The important point to be retained is that the volumetric estimates of subcortical structures when taken either bilaterally or unilaterally show similar statistical dependence.

\section{Discussion And Conclusions}

Imaging studies in the order of thousands of MRIs performed in the same center and using identical protocol and equipment are very costly. While it is possible to build aggregates of large datasets combining images from different imaging centers, issues related to the consistency of the results need to be carefully addressed. 


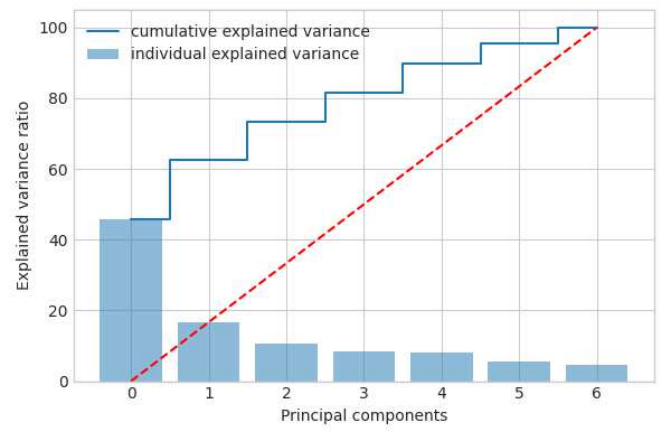

(a) Cumulative eigenvalue distribution for Left Hemisphere correlation matrix

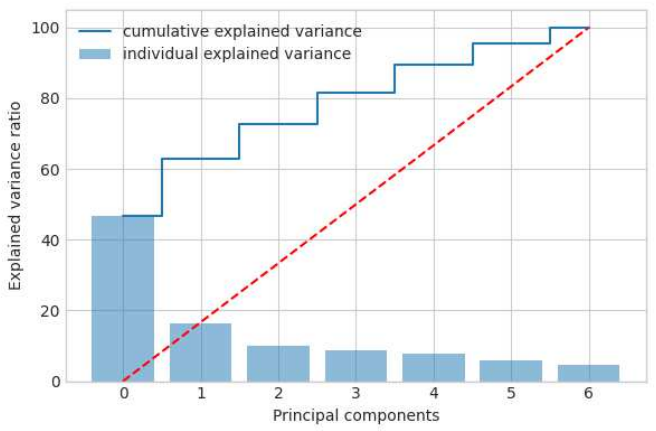

(b) Cumulative eigenvalue distribution for Right Hemisphere correlation matrix

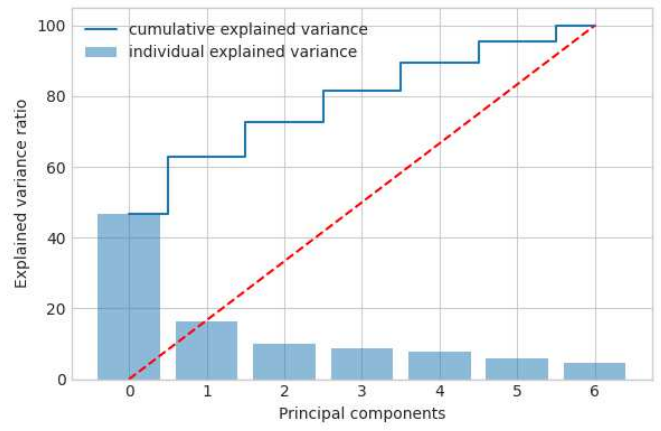

(c) Cumulative eigenvalue distribution for bilateral (Left vs Right structures) structures.

Figure 5: The eigenvalues explain the variance of the data along the eigenvectors (principal components). The figure shows the explained variance of each of the correlation matrices by depicting the cumulative eigenvalue (y-axis) versus the order of eigenvalues (x-axis). The explained variance can be calculated from the eigenvalues and tells us how much information (variance) can be attributed to each of the principal components. From the figure, we can deduce that the subcortical structures have the same statistical dependence when studied intrahemispherically (Figures 5b and 5c) and comparable with the interhemispherical eigenvalue analysis (Figure 5a)

We leverage a large single-center dataset of segmented subcortical structures to foster our understanding of the anatomic symmetric organization of the brain. Over 3,000 MRI scans obtained in the same center and using identical procedure were segmented to extract the volume of seven subcortical structures in the limbic system and the basal ganglia, namely thalamus, putamen, hippocampus, caudate, pallidum, amygdala, and accumbens. While several studies that compare variations in volume and alterations of symmetry in relation to the sexes, handedness [Ocklenburg and Gunturkun, 2012], [Kang et al., 2015], [Guadalupe et al., 2017], language |Corballis, 2009| or even as potential markers in brain disorders such as schizophrenia [Roalf et al., 2015], the study of the systemic volumetric interdependencies of subcortical structures has not received sufficient attention.

There is a large number of studies of sex differences in subcortical structures as well as studies that investigate the effect of ApolipoproteinE $\epsilon 4$ [Fleisher et al., 2005], [Tang et al., 2015] 
handedness [Szabó et al., 2003] and other factors on the volume of brain structures. We find no detectable effect on subcortical asymmetries when the sample is separated based on the allele $\epsilon 4$. Statistical tests found handedness-related asymmetries in the thalamus and hippocampus with no effect in accumbens and putamen. A recent meta-analysis [Guadalupe et al., 2017] did not find a significant effect of handedness on subcortical asymmetries, however, the same study found that asymmetry of certain structures eg. the putamen, vary with age. Finally, sex has a strong effect on the subcortical volumetric asymmetry in all structures except for the accumbens. This is in agreement with Bayesian hypothesis testing performed in 5216 participants in the UK Biobank [Ritchie et al., 2018] where no difference was found for the bilateral nucleus accumbens, finding, on the other hand, evidence for the hypothesis of a difference for all other regions.

The present study is interested in the characterization of the interdependency of subcortical structures in healthy, elderly brains. We propose a methodology based on Eigenvalue analysis to estimate the aggregate correlation of volumetric subcortical structures when they are studied in either the same hemisphere and in different hemispheres. While there is abundant literature in cortical and subcortical structural variation associated with biological [Eyler et al., 2011], [NicklJockschat et al., 2012], [Wen et al., 2016], [Bas-Hoogendam et al., 2018] and socioeconomic factors |Jenkins et al., 2020|, the effect of aging on both the intrahemispheric and the interhemispheric symmetry of subcortical structures is inadequately understood.

To our knowledge, this is the largest single-center study of subcortical global symmetry. We find that the seven structures studied have similar coupling when their volumes are studied either interhemispherically or intrahemispherically. The correlation matrix of all the structures segmented show that the statistical dependence for any given structure is always the largest with its twin structure in the other hemisphere. Along these lines, we could say that development is more important than hemispheric proximity.

Interhemispheric integration is inevitable for how the brain develops and grows [Gazzaniga, 2000|. Both hemispheric specialization and hemispheric integration are to be expected and are found in our results. By decomposing the correlation matrix into three submatrices containing the pairwise correlations both within the same hemisphere and in different hemispheres, we set apart three different views -left hemisphere, right hemisphere, and interhemispheric- of the statistical dependency between subcortical structures in the brain.

The aggregate statistical dependence for interhemispheric and intrahemispheric subcortical volume estimates are computed using two methods. First, directly from the correlation matrix via the $\mathrm{Z}$ inverse transform (Equation 3 ) and last, using Eigenvalue analysis to compute the cumulative curve of the distribution of the eigenvalues that would indicate the independence of the variables within each matrix. In either case, the statistical dependence is remarkably similar, indicating that the subcortical structures studied here have comparable coupling when taken as a whole for each hemisphere and when taken in different hemispheres.

The study overall indicates that anatomic bilateral symmetry is preserved in the aging human brain, supporting recent findings that postulate increased communication between distant brain areas as a mechanism to compensate for the deleterious effects of aging [Davis et al., 2017]. The characterization of brain subcortical symmetry proposed here allows new views of interhemispheric and intrahemispheric volume variation, setting the basis for future studies of anatomical symmetry and asymmetry in healthy brain aging.

\section{REFERENCES}

[Alaverdyan, 2019] Alaverdyan, Z. (2019). Unsupervised representation learning for anomaly detection on neuroimaging. Application to epilepsy lesion detection on brain MRI. PhD thesis, Universite de 
Lyon.

[Albin et al., 1989] Albin, R. L., Young, A. B., and Penney, J. B. (1989). The functional anatomy of basal ganglia disorders. Trends in neurosciences, 12(10):366-375.

[Bas-Hoogendam et al., 2018] Bas-Hoogendam, J. M., van Steenbergen, H., Tissier, R. L., HouwingDuistermaat, J. J., Westenberg, P. M., and van der Wee, N. J. (2018). Subcortical brain volumes, cortical thickness and cortical surface area in families genetically enriched for social anxiety disorder-a multiplex multigenerational neuroimaging study. EBioMedicine, 36:410-428.

[Breiman, 1996] Breiman, L. (1996). Bagging predictors. Machine learning, 24(2):123-140.

[Callen et al., 2001] Callen, D., Black, S. E., Gao, F., Caldwell, C., and Szalai, J. (2001). Beyond the hippocampus: Mri volumetry confirms widespread limbic atrophy in ad. Neurology, 57(9):16691674 .

[Chandola et al., 2009] Chandola, V., Banerjee, A., and Kumar, V. (2009). Anomaly detection: A survey. ACM computing surveys (CSUR), 41(3):1-58.

[Collier et al., 2003] Collier, D. C., Burnett, S. S., Amin, M., Bilton, S., Brooks, C., Ryan, A., Roniger, D., Tran, D., and Starkschall, G. (2003). Assessment of consistency in contouring of normal-tissue anatomic structures. Journal of applied clinical medical physics, 4(1):17-24.

[Corballis, 2009] Corballis, M. C. (2009). The evolution and genetics of cerebral asymmetry. Philosophical Transactions of the Royal Society B: Biological Sciences, 364(1519):867-879.

[Dale et al., 1999] Dale, A., Fischl, B., and Sereno, M. I. (1999). Cortical surface-based analysis: I. segmentation and surface reconstruction. NeuroImage, 9(2):179 - 194.

[Davis et al., 2017] Davis, S. W., Luber, B., Murphy, D. L., Lisanby, S. H., and Cabeza, R. (2017). Frequency-specific neuromodulation of local and distant connectivity in aging and episodic memory function. Human brain mapping, 38(12):5987-6004.

[Dennison et al., 2013] Dennison, M., Whittle, S., Yücel, M., Vijayakumar, N., Kline, A., Simmons, J., and Allen, N. B. (2013). Mapping subcortical brain maturation during adolescence: Evidence of hemisphere-and sex-specific longitudinal changes. Developmental science, 16(5):772-791.

[Desikan et al., 2006] Desikan, R. S., Ségonne, F., Fischl, B., Quinn, B. T., Dickerson, B. C., Blacker, D., Buckner, R. L., Dale, A. M., Maguire, R. P., Hyman, B. T., et al. (2006). An automated labeling system for subdividing the human cerebral cortex on mri scans into gyral based regions of interest. Neuroimage, 31(3):968-980.

[Despotović et al., 2015] Despotović, I., Goossens, B., and Philips, W. (2015). Mri segmentation of the human brain: challenges, methods, and applications. Computational and mathematical methods in medicine, 2015.

[Domingues et al., 2018] Domingues, R., Filippone, M., Michiardi, P., and Zouaoui, J. (2018). A comparative evaluation of outlier detection algorithms: Experiments and analyses. Pattern Recognition, 74:406-421.

[Esteves et al., 2019] Esteves, M., Moreira, P. S., Marques, P., Castanho, T. C., Magalhães, R., Amorim, L., Portugal-Nunes, C., Soares, J. M., Coelho, A., Almeida, A., et al. (2019). Asymmetrical subcortical plasticity entails cognitive progression in older individuals. Aging cell, 18(1):e12857. 
[Eyler et al., 2011] Eyler, L. T., Prom-Wormley, E., Fennema-Notestine, C., Panizzon, M. S., Neale, M. C., Jernigan, T. L., Fischl, B., Franz, C. E., Lyons, M. J., Stevens, A., et al. (2011). Genetic patterns of correlation among subcortical volumes in humans: results from a magnetic resonance imaging twin study. Human brain mapping, 32(4):641-653.

[Firbank et al., 2008] Firbank, M. J., Barber, R., Burton, E. J., and O'Brien, J. T. (2008). Validation of a fully automated hippocampal segmentation method on patients with dementia. Human brain mapping, 29(12):1442-1449.

[Fischl, 2012] Fischl, B. (2012). Freesurfer. Neuroimage, 62(2):774-781.

[Fischl et al., 2002] Fischl, B., Salat, D. H., Busa, E., Albert, M., Dieterich, M., Haselgrove, C., van der Kouwe, A., Killiany, R., Kennedy, D., Klaveness, S., Montillo, A., Makris, N., Rosen, B., and Dale, A. M. (2002). Whole brain segmentation: automated labeling of neuroanatomical structures in the human brain. Neuron, 33:341-355.

[Fischl et al., 1999] Fischl, B., Sereno, M. I., and Dale, A. (1999). Cortical surface-based analysis: Ii: Inflation, flattening, and a surface-based coordinate system. NeuroImage, 9(2):195 - 207.

[Fischl et al., 2004] Fischl, B., van der Kouwe, A., Destrieux, C., Halgren, E., SÃ@gonne, F., Salat, D. H., Busa, E., Seidman, L. J., Goldstein, J., Kennedy, D., Caviness, V., Makris, N., Rosen, B., and Dale, A. M. (2004). Automatically Parcellating the Human Cerebral Cortex. Cerebral Cortex, 14(1):11-22.

[Fjell et al., 2015] Fjell, A. M., Sneve, M. H., Storsve, A. B., Grydeland, H., Yendiki, A., and Walhovd, K. B. (2015). Brain Events Underlying Episodic Memory Changes in Aging: A Longitudinal Investigation of Structural and Functional Connectivity. Cerebral Cortex, page bhv102.

[Fleisher et al., 2005] Fleisher, A., Grundman, M., Jack, Clifford R., J., Petersen, R. C., Taylor, C., Kim, H. T., Schiller, D. H. B., Bagwell, V., Sencakova, D., Weiner, M. F., DeCarli, C., DeKosky, S. T., van Dyck, C. H., Thal, L. J., and Study, A. D. C. (2005). Sex, Apolipoprotein E e4 Status, and Hippocampal Volume in Mild Cognitive Impairment. Archives of Neurology, 62(6):953-957.

[Floris et al., 2020] Floris, D. L., Wolfers, T., Zabihi, M., Holz, N. E., Zwiers, M. P., Charman, T., Tillmann, J., Ecker, C., Dell'Acqua, F., Banaschewski, T., et al. (2020). Atypical brain asymmetry in autism-a candidate for clinically meaningful stratification. Biological Psychiatry: Cognitive Neuroscience and Neuroimaging.

[FreeSurfer cortical reconstruction and parcellation process., 2017] FreeSurfer cortical reconstruction and parcellation process. (2017). Anatomical processing script: recon-all. https: //surfer.nmr.mgh.harvard.edu/fswiki/recon-all, Last accessed on 2020-15-30.

[Gazzaniga, 2000] Gazzaniga, M. S. (2000). Cerebral specialization and interhemispheric communication: does the corpus callosum enable the human condition? Brain, 123(7):1293-1326.

[Giannakopoulos et al., 1994] Giannakopoulos, P., Bouras, C., and Hof, P. R. (1994). Alzheimer's disease with asymmetric atrophy of the cerebral hemispheres: morphometric analysis of four cases. Acta neuropathologica, 88(5):440-447.

[Gómez-Ramírez et al., 2020] Gómez-Ramírez, J., Ávila-Villanueva, M., and Fernández-Blázquez, M. Á. (2020). Selecting the most important self-assessed features for predicting conversion to mild cognitive impairment with random forest and permutation-based methods. Scientific Reports, 10(1):1-15. 
[Guadalupe et al., 2017] Guadalupe, T., Mathias, S. R., Theo, G., Whelan, C. D., Zwiers, M. P., Abe, Y., Abramovic, L., Agartz, I., Andreassen, O. A., Arias-Vásquez, A., et al. (2017). Human subcortical brain asymmetries in 15,847 people worldwide reveal effects of age and sex. Brain imaging and behavior, 11(5):1497-1514.

[Gunbey et al., 2014] Gunbey, H. P., Ercan, K., Findıkoglu, A. S., Bulut, H. T., Karaoglanoglu, M., and Arslan, H. (2014). The limbic degradation of aging brain: a quantitative analysis with diffusion tensor imaging. The Scientific World Journal, 2014.

[Herron et al., 2015] Herron, T. J., Kang, X., and Woods, D. L. (2015). Sex differences in cortical and subcortical human brain anatomy. F1000Research, 4(88):88.

[Hervé et al., 2013] Hervé, P.-Y., Zago, L., Petit, L., Mazoyer, B., and Tzourio-Mazoyer, N. (2013). Revisiting human hemispheric specialization with neuroimaging. Trends in cognitive sciences, 17(2):69-80.

[Jenkins et al., 2020] Jenkins, L. M., Chiang, J. J., Vause, K., Hoffer, L., Alpert, K., Parrish, T. B., Wang, L., and Miller, G. E. (2020). Subcortical structural variations associated with low socioeconomic status in adolescents. Human Brain Mapping, 41(1):162-171.

[Kang et al., 2015] Kang, X., Herron, T. J., Ettlinger, M., and Woods, D. L. (2015). Hemispheric asymmetries in cortical and subcortical anatomy. Laterality: Asymmetries of Body, Brain and Cognition, 20(6):658-684.

[Kong et al., 2020] Kong, X.-Z., Postema, M. C., Guadalupe, T., de Kovel, C., Boedhoe, P. S., Hoogman, M., Mathias, S. R., Van Rooij, D., Schijven, D., Glahn, D. C., et al. (2020). Mapping brain asymmetry in health and disease through the enigma consortium. Human Brain Mapping.

[Kreitzer and Malenka, 2008] Kreitzer, A. C. and Malenka, R. C. (2008). Striatal plasticity and basal ganglia circuit function. Neuron, 60(4):543-554.

[Langsrud, 2003] Langsrud, Ø. (2003). Anova for unbalanced data: Use type ii instead of type iii sums of squares. Statistics and Computing, 13(2):163-167.

[Lashley, 1958] Lashley, K. S. (1958). Cerebral organization and behavior. Research Publications of the Association for Research in Nervous \& Mental Disease.

[LeDoux et al., 2020] LeDoux, J. E., Michel, M., and Lau, H. (2020). A little history goes a long way toward understanding why we study consciousness the way we do today. Proceedings of the National Academy of Sciences, 117(13):6976-6984.

[Leonard and Eckert, 2008] Leonard, C. M. and Eckert, M. A. (2008). Asymmetry and dyslexia. Developmental neuropsychology, 33(6):663-681.

[Liu et al., 2008] Liu, F. T., Ting, K. M., and Zhou, Z.-H. (2008). Isolation forest. In 2008 Eighth IEEE International Conference on Data Mining, pages 413-422. IEEE.

[McLachlan, 2009] McLachlan, R. S. (2009). A brief review of the anatomy and physiology of the limbic system. Canadian Journal of Neurological Sciences, 36.

[Morillon et al., 2010] Morillon, B., Lehongre, K., Frackowiak, R. S., Ducorps, A., Kleinschmidt, A., Poeppel, D., and Giraud, A.-L. (2010). Neurophysiological origin of human brain asymmetry for speech and language. Proceedings of the National Academy of Sciences, 107(43):18688-18693. 
[Narvacan et al., 2017] Narvacan, K., Treit, S., Camicioli, R., Martin, W., and Beaulieu, C. (2017). Evolution of deep gray matter volume across the human lifespan. Human brain mapping, 38(8):3771-3790.

[Neubauer et al., 2020] Neubauer, S., Gunz, P., Scott, N. A., Hublin, J.-J., and Mitteroecker, P. (2020). Evolution of brain lateralization: A shared hominid pattern of endocranial asymmetry is much more variable in humans than in great apes. Science advances, 6(7):eaax9935.

[Nickl-Jockschat et al., 2012] Nickl-Jockschat, T., Kleiman, A., Schulz, J. B., Schneider, F., Laird, A. R., Fox, P. T., Eickhoff, S. B., and Reetz, K. (2012). Neuroanatomic changes and their association with cognitive decline in mild cognitive impairment: a meta-analysis. Brain Structure and Function, 217(1):115-125.

[Nielsen et al., 2013] Nielsen, J. A., Zielinski, B. A., Ferguson, M. A., Lainhart, J. E., and Anderson, J. S. (2013). An evaluation of the left-brain vs. right-brain hypothesis with resting state functional connectivity magnetic resonance imaging. PloS one, 8(8):e71275.

[Núñez et al., 2018] Núñez, C., Theofanopoulou, C., Senior, C., Cambra, M. R., Usall, J., StephanOtto, C., and Brébion, G. (2018). A large-scale study on the effects of sex on gray matter asymmetry. Brain Structure and Function, 223(1):183-193.

[Ocklenburg and Gunturkun, 2012] Ocklenburg, S. and Gunturkun, O. (2012). Hemispheric asymmetries: the comparative view. Frontiers in psychology, 3:5.

[Okada et al., 2018] Okada, N., Yahata, N., Koshiyama, D., Morita, K., Sawada, K., Kanata, S., Fujikawa, S., Sugimoto, N., Toriyama, R., Masaoka, M., et al. (2018). Abnormal asymmetries in subcortical brain volume in early adolescents with subclinical psychotic experiences. Translational psychiatry, 8(1):1-11.

[Palmer, 2004] Palmer, A. R. (2004). Symmetry breaking and the evolution of development. Science, 306(5697):828-833.

[Pedregosa et al., 2011] Pedregosa, F., Varoquaux, G., Gramfort, A., Michel, V., Thirion, B., Grisel, O., Blondel, M., Prettenhofer, P., Weiss, R., Dubourg, V., Vanderplas, J., Passos, A., Cournapeau, D., Brucher, M., Perrot, M., and Duchesnay, E. (2011). Scikit-learn: Machine learning in Python. Journal of Machine Learning Research, 12:2825-2830.

[Rane et al., 2017] Rane, S., Plassard, A., Landman, B. A., Claassen, D. O., and Donahue, M. J. (2017). Comparison of cortical and subcortical measurements in normal older adults across databases and software packages. Journal of Alzheimer's disease reports, 1(1):59-70.

[Ritchie et al., 2018] Ritchie, S. J., Cox, S. R., Shen, X., Lombardo, M. V., Reus, L. M., Alloza, C., Harris, M. A., Alderson, H. L., Hunter, S., Neilson, E., et al. (2018). Sex differences in the adult human brain: evidence from 5216 uk biobank participants. Cerebral Cortex, 28(8):2959-2975.

[Roalf et al., 2015] Roalf, D. R., Vandekar, S. N., Almasy, L., Ruparel, K., Satterthwaite, T. D., Elliott, M. A., Podell, J., Gallagher, S., Jackson, C. T., Prasad, K., et al. (2015). Heritability of subcortical and limbic brain volume and shape in multiplex-multigenerational families with schizophrenia. Biological psychiatry, 77(2):137-146.

[Ruigrok et al., 2014] Ruigrok, A. N., Salimi-Khorshidi, G., Lai, M.-C., Baron-Cohen, S., Lombardo, M. V., Tait, R. J., and Suckling, J. (2014). A meta-analysis of sex differences in human brain structure. Neuroscience \& Biobehavioral Reviews, 39:34-50. 
[Seabold and Perktold, 2010] Seabold, S. and Perktold, J. (2010). statsmodels: Econometric and statistical modeling with python. In 9th Python in Science Conference.

[Shaw and Alvord Jr, 1997] Shaw, C.-M. and Alvord Jr, E. C. (1997). Neuropathology of the limbic system. Neuroimaging Clinics of North America, 7(1):101-142.

[Sperry, 1961] Sperry, R. W. (1961). Cerebral organization and behavior. Science, 133(3466):17491757.

[Sperry, 1984] Sperry, R. W. (1984). Consciousness, personal identity and the divided brain. Neuropsychologia.

[Szabó et al., 2003] Szabó, C. Á., Lancaster, J. L., Xiong, J., Cook, C., and Fox, P. (2003). Mr imaging volumetry of subcortical structures and cerebellar hemispheres in normal persons. American Journal of Neuroradiology, 24(4):644-647.

[Tang et al., 2015] Tang, X., Holland, D., Dale, A. M., and Miller, M. I. (2015). Apoe affects the volume and shape of the amygdala and the hippocampus in mild cognitive impairment and alzheimer's disease: age matters. Journal of Alzheimer's Disease, 47(3):645-660.

[van der Knaap and van der Ham, 2011] van der Knaap, L. J. and van der Ham, I. J. (2011). How does the corpus callosum mediate interhemispheric transfer? a review. Behavioural brain research, 223(1):211-221.

[Vos et al., 2019] Vos, M., Starmans, M., Timbergen, M., van der Voort, S., Padmos, G., Kessels, W., Niessen, W., van Leenders, G., Grünhagen, D., Sleijfer, S., et al. (2019). Radiomics approach to distinguish between well differentiated liposarcomas and lipomas on mri. The British journal of surgery, 106(13):1800.

[Wen et al., 2016] Wen, W., Thalamuthu, A., Mather, K. A., Zhu, W., Jiang, J., De Micheaux, P. L., Wright, M. J., Ames, D., and Sachdev, P. S. (2016). Distinct genetic influences on cortical and subcortical brain structures. Scientific reports, 6(1):1-11.

[Willems et al., 2010] Willems, R. M., Peelen, M. V., and Hagoort, P. (2010). Cerebral lateralization of face-selective and body-selective visual areas depends on handedness. Cerebral cortex, 20(7):1719-1725. 


\section{Data ANd Code AVAIlability}

The data set can be downloaded from https://github.com/grjd/bilateralbrain .

\section{ACKNOWLEDGEMENTS}

The authors would like to thank the generous persons that volunteered to participate in the study and Fundación Reina Sofía for their support. The authors acknowledge funding from Ministerio de Ciencia, Innovación y Universidades (CONNECT-AD) RTI2018-098762-B-C31 and Structural Funds ERDF (INTERREG V-A Spain-Portugal (POCTEP) Grant: 0348CIE6E).

\section{ETHICS DECLARATIONS}

The authors declare no competing interests. 


\section{Figures}

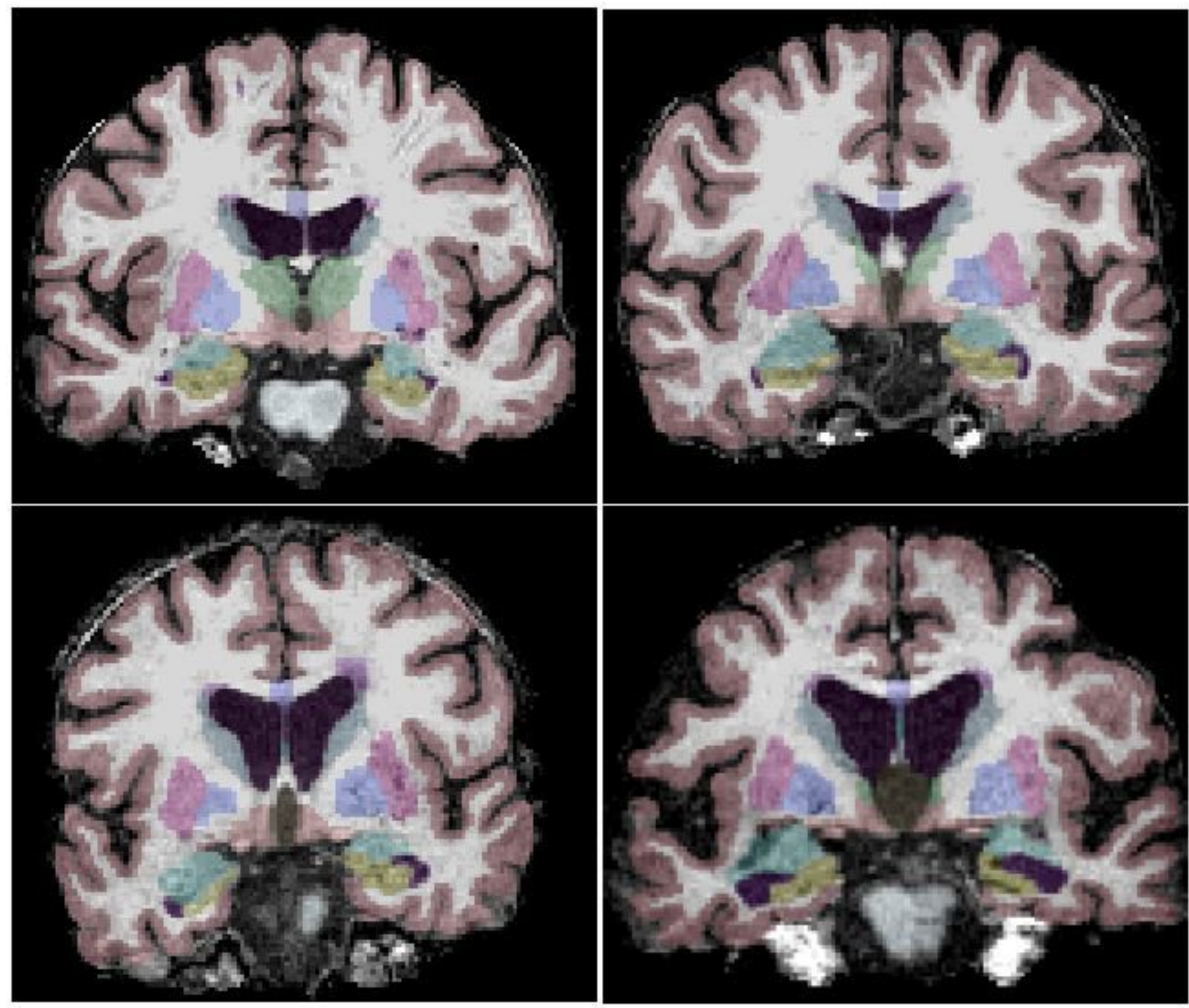

Figure 1

Subcortical structures are clusters of cell bodies, buried in white matter area. Coronal view of the subcortical segmentation realized in 4 different subjects in the study. Clockwise from top left: 75 female 75, 74 male, 76 female, 83 female. 


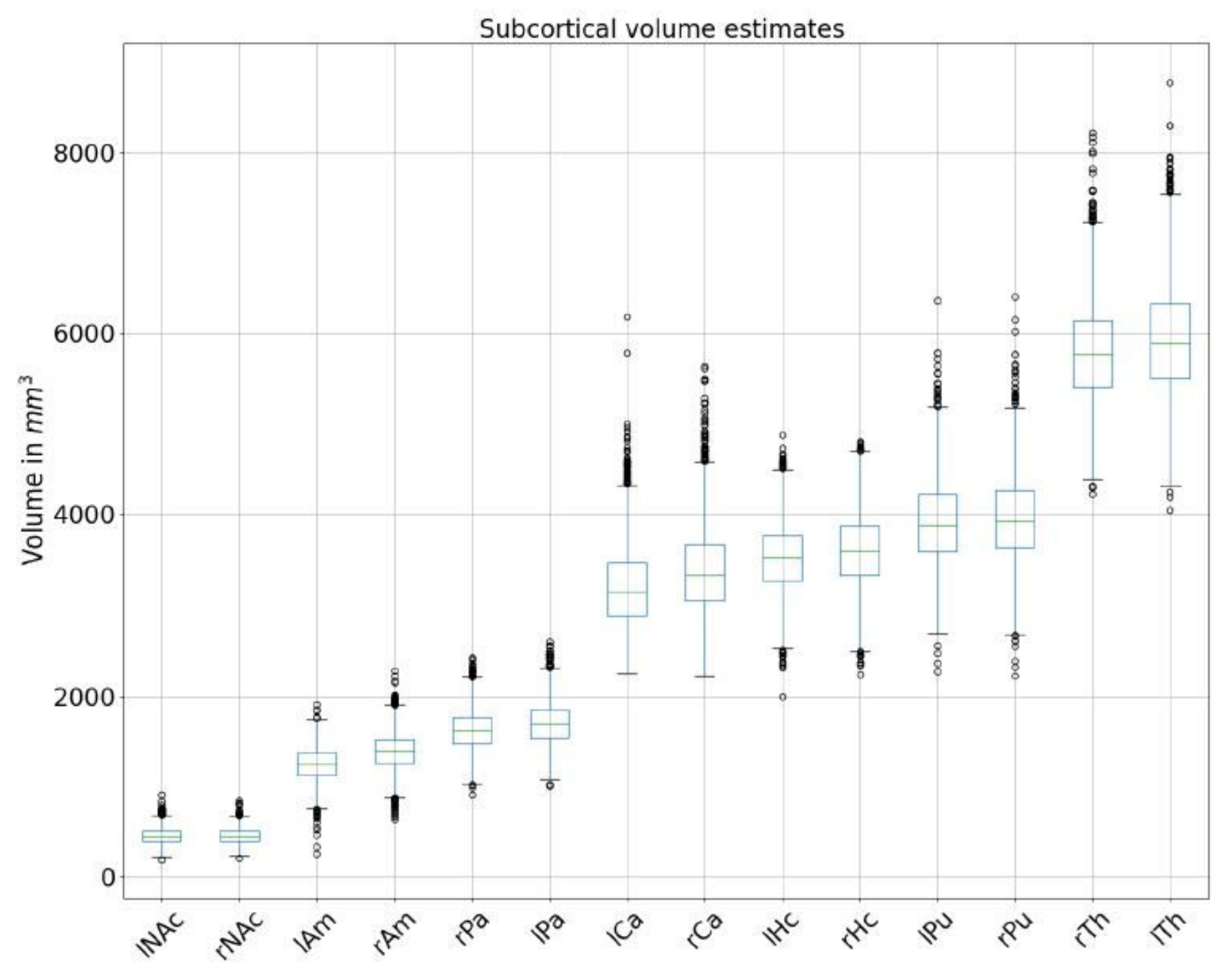

Figure 2

Boxplot of the 7 bilateral subcortical structures segmented with FreeSurfer Healthy subjects only. The estimated median volumes from the smallest to the largest structure is as follows: Accumbens R/L 459.10/457.10mm3, Amygdala R/L 1399.31/1252.65mm3, Pallidum R/L 1632.85/1700.82mm3, Caudate R/L 3379.04/3202.93mm3, Hippocampus R/L 3312.00/3312.00mm3, Putamen R/L 3952.21/3916.95mm3, Thalamus R/L 5798.49/5946.30mm3 


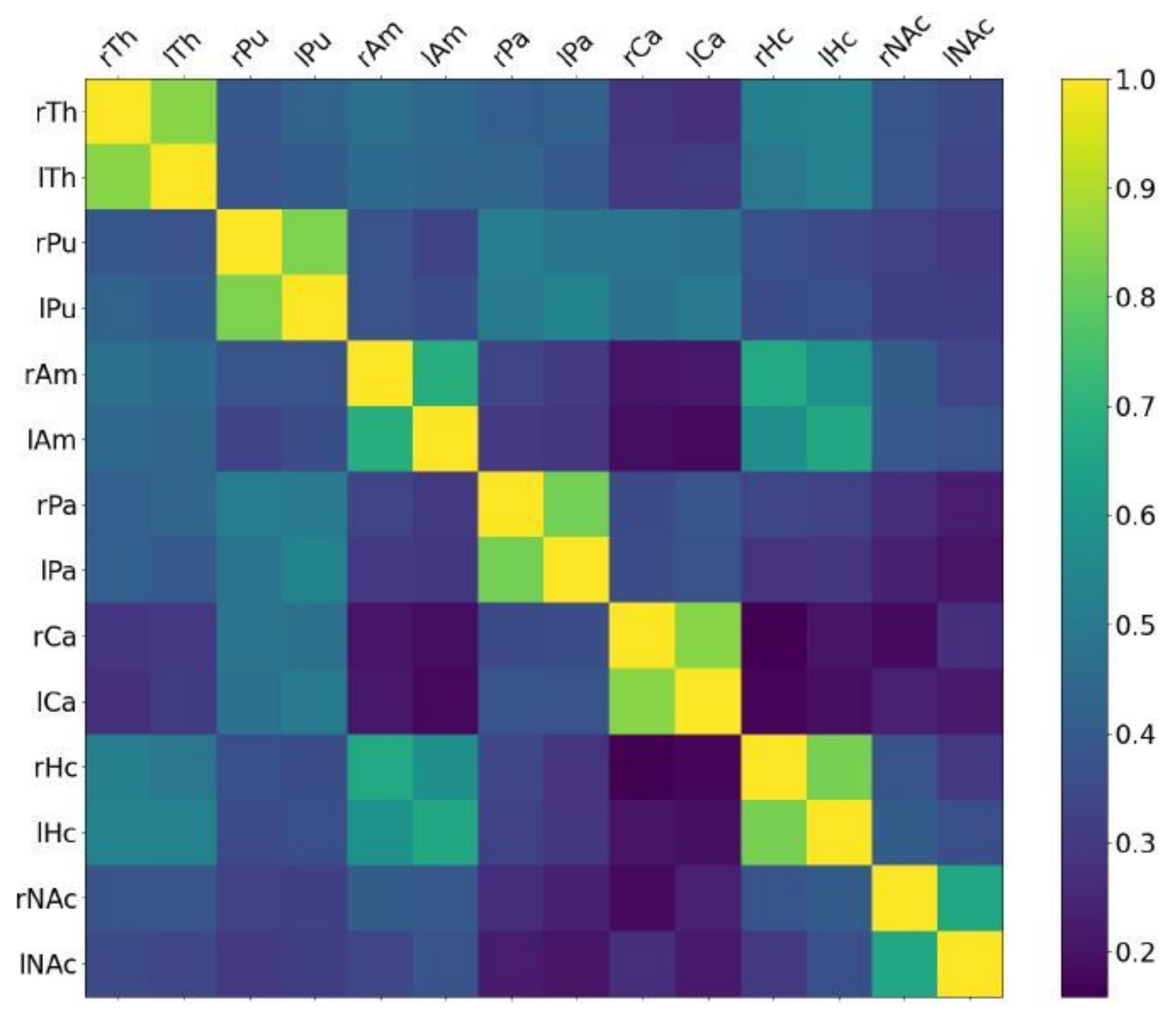

\section{Figure 3}

Correlation matrix of the 7 bilateral subcortical structures segmented with FreeSurfer. The elements of the diagonal are naturally 1 , the correlation matrix seems to indicate that the strongest statistical dependence is for the inter hemispheric correlation of the same structure. 


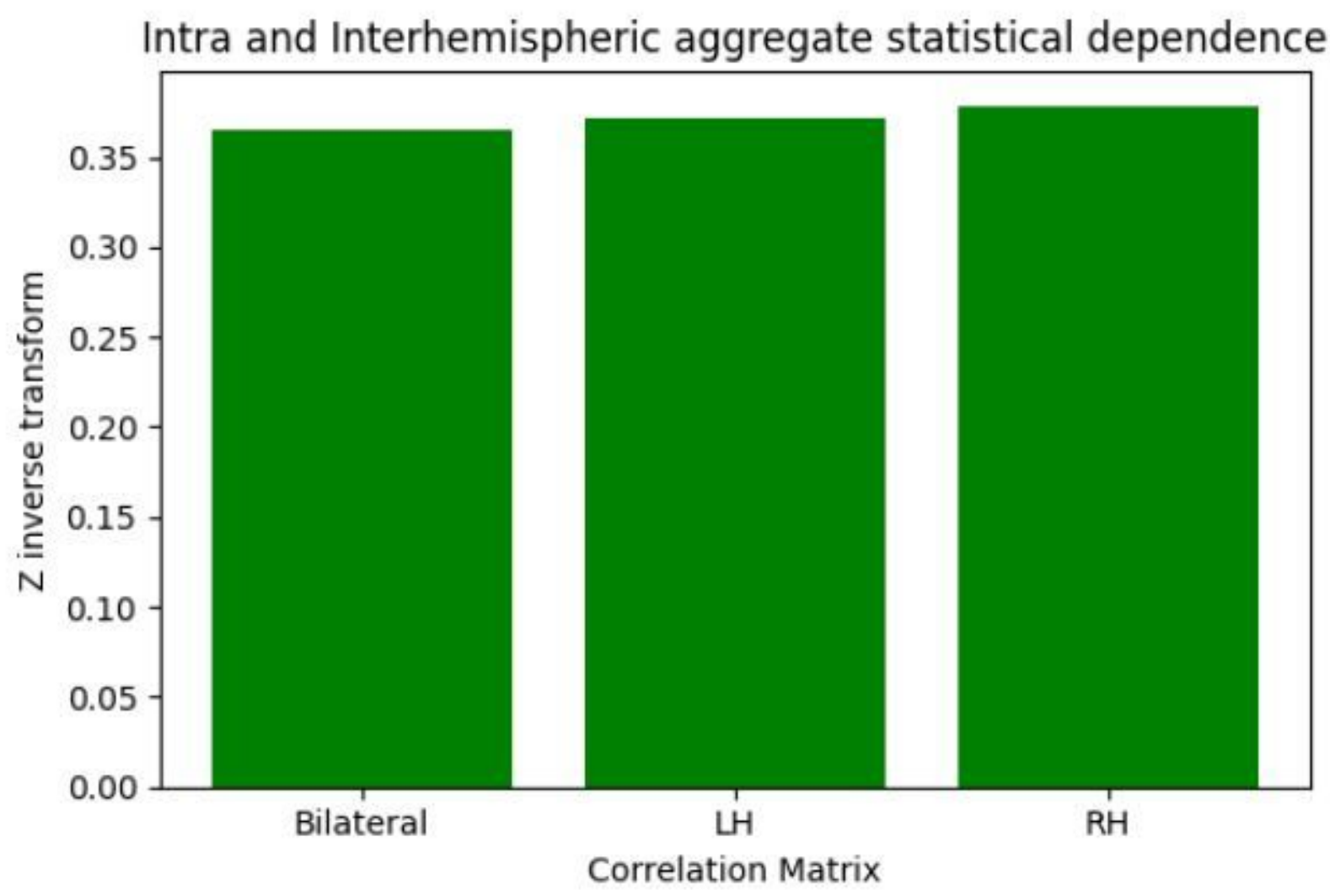

Figure 4

Aggregate statistical dependence for interhemispheric and intrahemispheric subcortical volume estimates computed with the $Z$ inverse transform (Equation 3 ). The statistical dependence is remarkably similar in all three cases. Of note, the main diagonal has been excluded from the computation of the $Z$ transform to avoid biased towards the interhemispheric matrices which symmetric and have 1 in the main diagonal which is not the case in the Bilateral correlation matrix. 


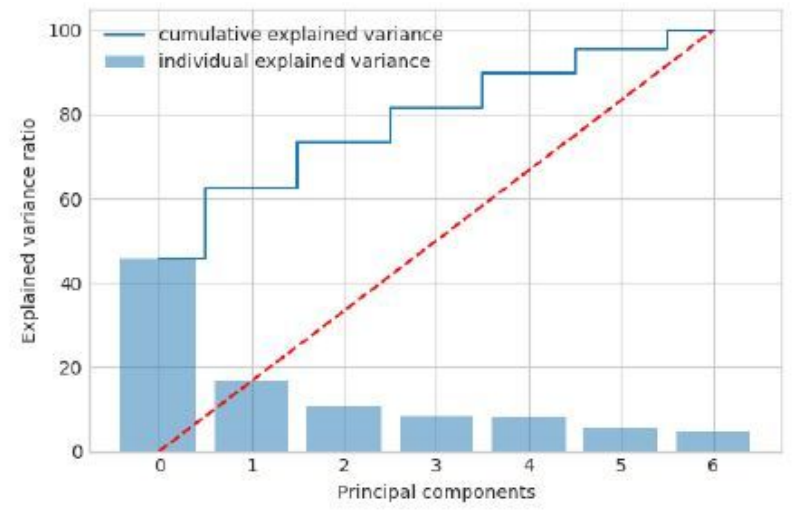

(a) Cumulative eigenvalue distribution for Left Hemisphere correlation matrix

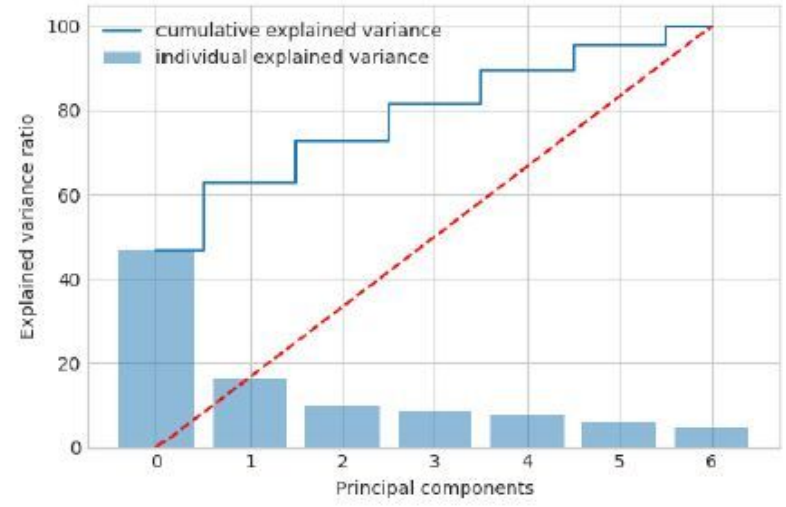

(b) Cumulative eigenvalue distribution for Right Hemisphere correlation matrix

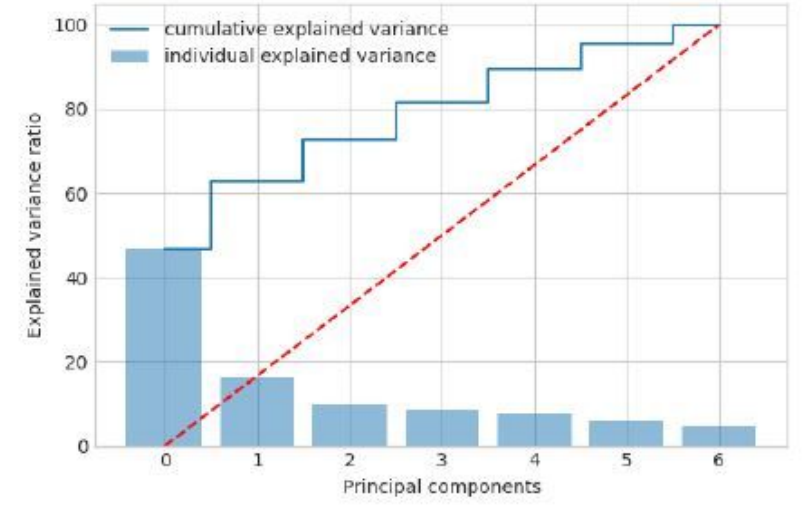

(c) Cumulative eigenvalue distribution for bilateral (Left vs Right structures) structures.

\section{Figure 5}

The eigenvalues explain the variance of the data along the eigenvectors (principal components). The figure shows the explained variance of each of the correlation matrices by depicting the cumulative eigenvalue (y-axis) versus the order of eigenvalues (x-axis). The explained variance can be calculated from the eigenvalues and tells us how much information (variance) can be attributed to each of the principal components. From the figure, we can deduce that the subcortical structures have the same statistical dependence when studied intrahemispherically (Figures $5 b$ and $5 c$ ) and comparable with the interhemispherical eigenvalue analysis (Figure 5a) 